\title{
Vom „Voneinander Lernen“ zum „Gemeinsam Wirken“ - die strategische Weiterentwicklung des ddn
}

\author{
S. Fassio, M. Schmeink und C. Zeckra
}

\section{Einleitung}

Einhergehend mit der Erkenntnis, dass ab dem Jahr 2003 in Deutschland die Bevölkerungszahlen kontinuierlich abnehmen würden, wurde $a b$ der Jahrtausendwende in Deutschland der demografische Wandel erstmals als eine konkrete Herausforderung für die Gesellschaft identifiziert (Statistisches Bundesamt 2006, S. 5). Die Bedrohung, als die der demografische Wandel vor allem mit Blick auf die Arbeitswelt und die Rentenversorgung verstanden wurde, hat sich heute, nach einer fast zwanzigjährigen Auseinandersetzung mit der Thematik, in ein ganzheitlicheres Verständnis verwandelt. Lag zu Beginn der Fokus von Wissenschaft, Politik und Wirtschaft verstärkt auf den alternden Belegschaften von Betrieben, so ist heute längst ersichtlich geworden, dass uns der demografische Wandel vor ein breites Spektrum komplexer gesamtgesellschaftlicher Herausforderungen stellt - und dass der Aspekt „Alter" nur einer von vielen ist. Diese Aspekte beinhalten humane Faktoren, wie beispielsweise der Umgang mit multikulturellen Belegschaften in international ausgerichteten Unternehmen oder die Integration von Zuwanderern in kleinen oder mittelständischen Betrieben. Gleichzeitig zeigt ein Blick auf unsere Gesellschaft auch, dass sich die Formulierung individueller Lebensentwürfe verstärkt durchsetzt, sich Geschlechter von klassischen Rollenbildern loslösen und sich somit auch Ansprüche an die eigene Arbeit wie auch die Familienplanung verändern - ein Prozess, der sicherlich noch lange nicht abgeschlossen ist.

Im Umkehrschluss stellen auch Unternehmen immer höhere Ansprüche an ihre Beschäftigten. An diesem Punkt nimmt die digitale Transformation unserer Gesellschaft eine Schlüsselfunktion ein, da sie den demografischen Wandel nun auch um eine technologische Dimension erweitert. Die Digitalisierungsschübe hin zu einer Industrie 4.0 machen vertrautes Wissen an vielen Stellen obsolet und erfordern ein Neudenken des Systems Arbeit. Die Verbindung der physikalischen Welt mit dem „Internet der Dinge“" (Botthof \& Hartmann 2015, Vorwort) macht Science-Fiction zur neuen Realität, die Mensch-Maschine-Interaktion zum Arbeitsalltag 
und den Umgang mit Algorithmen und Künstlicher Intelligenz zur Arbeitswelt des 21. Jahrhunderts.

Was bedeutet dies nun konkret für Unternehmen und Beschäftigte? Zuerst einmal lässt sich sagen, dass der demografische Wandel eine Herausforderung ist, die sich gesamtgesellschaftlich stellt und konsequenterweise nicht ausschließlich im Unternehmenskontext bewältigt werden kann. Nur im Zusammenspiel aller Akteure können erfolgreiche Maßnahmen zur Bearbeitung dieses Phänomens entwickelt und implementiert werden, die nicht nur problemzentriert ausgelegt sind, sondern den demografischen Wandel auch als Initiator für neue Impulse sehen. Für die Personalarbeit von Unternehmen des 21. Jahrhunderts bedeutet dies, dass nicht mehr nur der eigene unternehmerische Erfolg zählt, sondern auch die eigene Rolle als Mitglied einer sozialen, demokratischen Gesellschaft erkannt und Verantwortung für den zuvor genannten demografischen Wertewandel übernommen werden sollte - ein Prozess, für den unternehmensübergreifender Austausch und Netzwerkarbeit unabdingbar sind.

Der folgende Beitrag soll sich deshalb mit der Frage befassen, inwiefern (Unternehmens-)Netzwerke dazu beitragen können, konkrete Fragestellungen des demografischen Wandels praxisorientiert zu beantworten und welcher Voraussetzungen es hierfür bedarf. Untersuchungsgegenstand ist das Unternehmensnetzwerk Das Demographie Netzwerk e.V. (ddn), welches sich bereits seit über 14 Jahren mit den Themen des demografischen Wandels beschäftigt und sich - parallel dazu - kontinuierlich weiterentwickelt hat.

\section{Das Demographie Netzwerk e.V. als Impulsgeber}

Das Demographie Netzwerk e.V. (ddn) wurde im Frühjahr 2006 als ein Unternehmensnetzwerk der Initiative Neue Qualität der Arbeit (INQA) gegründet und versteht sich als ein Impulsgeber"in zu den komplexen Herausforderungen des demografischen Wandels für unsere Gesellschaft. Mit der Gründung des ddn wurde bereits in der Gründungsveranstaltung die klare Vision des Netzwerks definiert, nämlich „zukunftsfähige erfolgreiche Beschäftigungsstrategien in Unternehmen unter den Bedingungen des demografischen Wandels" (Anzinger 2006) zu entwickeln.

Der gemeinnützige Verein agiert dabei auf dem gesamten Bundesgebiet und richtet sich nicht nur an Unternehmen und KMU, sondern auch an Akteure aus Zivilgesellschaft, Wissenschaft sowie Verwaltung. Bereits daraus lässt sich der gesamtgesellschaftliche Ansatz nachvollziehen, der dem Netzwerk zugrunde liegt und konsequent in der Vereinsarbeit verfolgt 
wird. Der unternehmens- bzw. branchenübergreifende Austausch ist das zentrale Instrument des Vereins, um diskursive Prozesse zu initiieren und äußert sich neben zahlreichen Veranstaltungsangeboten auch in der Gründung von über 13 Arbeitskreisen sowie weiteren 17 Regionalnetzwerken. Doch ist das Selbstverständnis der Vereinsarbeit nicht nur auf die interne Vereinsarbeit konzentriert, sondern will auch über die eigenen Grenzen hinaus aktiv werden. Zu diesem Zweck wurde der Deutsche Demografie Preis ins Leben gerufen, der 2020 erstmals stattfinden konnte. In Zusammenarbeit mit weiteren Kooperationspartnern werden durch die Vergabe des Preises Projekte ausgezeichnet, die sich in der Bewältigung des demografischen Wandels gesellschaftlich besonders hervorheben. Diesen soll damit eine Vorbildrolle zugewiesen werden, die andere Betriebe ebenfalls dazu motiviert, sich dem demografischen Wandel anzunehmen.

Ähnlich, wie sich die Entwicklung des demografischen Wandels vollzogen hat, so lässt sich auch der Ansatz der Vereinsarbeit als chronologischer Prozess darstellen, der sich im Laufe der letzten 14 Jahre immer weiter an aktuelle Entwicklungen angepasst hat. Der Fokus liegt dabei stets auf einer zukunftsgerichteten Personalpolitik von Betrieben, die soziale, technologische wie auch wirtschaftliche Aspekte miteinander verknüpft.

Vor allem in der Praxis, also im konkreten Unternehmensalltag, ist die Perspektive auf Personalpolitik zumeist nach innen ausgerichtet, d.h. die Bedürfnisse der Unternehmen werden in den Vordergrund gestellt (Marquardt et al. 2019, S. 15). Zu Beginn der 2000er-Jahre bedeutete dies in erster Linie die Sicherung der Beschäftigungsfähigkeit der Angestellten im Beruf, beispielsweise durch Maßnahmen zur Gesundheitsförderung bis hin zu einem umfassenden Gesundheitsmanagement. Daran lässt sich schnell ablesen, dass Beschäftigte von Unternehmen weniger als Individuen, sondern vielmehr als eine Ressource verstanden wurden (ebd., S. 16), die vordergründig zur Steigerung der unternehmerischen Leistungskraft diente und deshalb "gesichert" werden musste. Dieses veraltete Bild zur Personalarbeit war der Startschuss für die Netzwerkarbeit des ddn, denn es galt, diese alten Strukturen aufzubrechen und durch neue zu ersetzen.

Gerade in der Weiterentwicklung der unternehmerischen Personalpolitik spielen Netzwerke eine zentrale Rolle, denn in diesen Zusammenschlüssen findet ein aktiver Diskurs statt, der die nach innen gerichtete Perspektive von Unternehmen aufzubrechen vermag. Hier kann im sicherem Raum Personalarbeit um- bzw. neugedacht werden.

Der unternehmensübergreifende Austausch kann sich dabei in der Diskussion ganz konkreter Ziele äußern, beispielsweise in der Identifizierung strategischer Maßnahmen zum Employer Branding, die dafür Sorge tragen sollen, dass die individuellen Bedürfnisse der Mitarbeitenden angespro- 
chen werden. Auf der anderen Seite eignen Netzwerke sich jedoch auch dazu, neue Methoden anzuwenden, die es erlauben, die Perspektive der $\mathrm{Zi}$ vilgesellschaft in die Personalarbeit einzubeziehen (Marquardt et al. 2019, S. 15-16).

Vor allem unter der Berücksichtigung von Best-Practice-Beispielen oder des Konzepts der kollegialen Beratung werden im ddn aktiv Lösungsstrategien und Denkanstöße zur Formulierung von neuen Konzepten herangezogen. Das Prinzip der Best-Practices untersucht dabei erfolgreiche Beispiele aus der Praxis mit Blick auf deren Allgemeingültigkeit und Übertragbarkeit auf andere Bereiche und Unternehmen. Die Idee dahinter ist ein „Voneinander Lernen“, die gerade für kleinere und/oder noch junge Unternehmen eine Hilfestellung sein kann, um sich zu orientieren. Gleichzeitig sollte das Best-Practice-Prinzip auch kritisch betrachtet werden, da Übertragbarkeit zumeist nur in Grenzen möglich ist und das simple „Kopieren" bestimmter Maßnahmen aus dem jeweiligen Fallbeispiel nicht automatisch mit größerem Erfolg gekrönt sein muss. Denn nicht nur jede" $r$ Mitarbeitende in einem Unternehmen hat individuelle Bedürfnisse und Voraussetzungen, sondern auch jedes Unternehmen hat seine Spezifika.

\section{Das Prinzip der kollegialen Beratung}

In diesem Zuge hat sich das Konzept der kollegialen Beratung bzw. des kollegialen Benchmarkings etabliert. Dieser Kommunikationsansatz hat sich zum primären Instrument des ddn entwickelt und spiegelt sich vor allem in den Arbeitskreisen des Netzwerks wider. Das kollegiale Benchmarking versteht sich dabei als ein Gegenentwurf zum betriebsinternen Finden von Lösungen und bezieht die externe Perspektive bzw. Beratung mit ein, um so von anderen zu lernen, externe Konzepte aber nicht 1:1 zu kopieren. Dabei geht es vor allem um den Austausch zwischen Fachkolleg*innen und die Erweiterung der eigenen Perspektive auf spezifische Unternehmensaspekte. Fleck und Krauß beschrieben diesen Ansatz wie folgt: „Bei dieser Methode des systematischen Vergleichens zwischen internen Abteilungen oder externen Benchmark-Partnern ist es das Ziel, Optimierungspotenziale $z u$ erkennen und die jeweils besten Lösungen miteinander zu verbinden" (Fleck \& Krauß 2011, S. 31). Das Prinzip bietet den großen Vorteil, dass es auch oder gerade dann - greift, wenn keine oder nur wenige Bezugsreferenzen vorliegen. Strategien zur Bewältigung des demografischen Wandels in Unternehmen eignen sich deshalb besonders für diese Methode, da sie sich zumeist erst über einen mittel- bis langfristigen Zeitraum bemerkbar machen. Im Rahmen der Methode des kollegialen Benchmarkings werden je- 
doch zumeist nur Teillösungen oder noch nicht abgeschlossene Projekte analysiert und ggf. optimiert. Das Ziel dabei ist nicht etwa die ganzheitliche Lösung, sondern vielmehr das Finden von Lösungsansätzen oder -fragmenten, die der Optimierung einer Maßnahme oder Strategie dienen (ebd., S. 31-33).

Mit zunehmender Entwicklung dieser und ähnlicher Ansätze hat sich innerhalb der Unternehmen die Erkenntnis verbreitet, dass es von großem Nutzen ist, in den unternehmensübergreifenden Austausch zu treten und sich ggf. auch selbst zu organisieren. Diese Tendenz wurde auch seitens der Politik gespiegelt, indem gezielt Maßnahmen zum selbstorganisierten Austausch von Unternehmen gefördert wurden. Gerade für Kleinstunternehmen sowie kleine und mittlere Unternehmen (KMU) sollte so eine Austausch-Plattform für zukunftsgerichtete Personalpolitik und Unternehmensentwicklung eingerichtet werden.

Aus ebendiesem Impuls heraus wurde im Frühjahr 2006 ddn gegründet und verfolgt seitdem einen nationalen wie auch regionalen Ansatz. BestPractices wie auch kollegiales Benchmarking dominieren den Diskurs innerhalb der Netzwerkpartner, zeigen diesen jedoch auch mit Fortschreiten des demografischen Wandels gleichzeitig die eigenen Grenzen auf. Nicht zuletzt die Corona-Pandemie, die auch das Netzwerk seit März 2020 vor ungekannte Herausforderungen gestellt hat, verdeutlicht, dass selbst ein unternehmens- und branchenübergreifender Austausch nicht allen Fragen standhalten kann. So setzt sich ein immer ganzheitlicheres Konzept durch, das die Angebote und Ausrichtung des ddn zunehmend definiert, nämlich das sogenannte Collective Impact-Prinzip.

\section{Der Collective Impact: Personalarbeit zwischen Unternehmen und Gesellschaft}

Bereits in der Einführung dieses Beitrages wurde die Wechselwirkung zwischen gesellschaftlichen und unternehmerischen Veränderungsprozessen erwähnt und soll im Folgenden noch ausführlicher besprochen werden. Für das 21. Jahrhundert wurden von der Wissenschaft sogenannte „Megatrends" formuliert, mit denen sich die Gesellschaft konfrontiert sieht, und zu denen neben der Digitalisierung u.a. auch die Neue Mobilität und der demografische Wandel gehört (RAG Stiftung). Bereits die Begrifflichkeit des Megatrends legt nahe, dass es sich hier um kein kurzzeitig auftretendes Phänomen handelt, sondern um einen Transformationsprozess, der alle gesellschaftlichen Akteure einbezieht. So entwickelte sich das 
Prinzip des „Collective Impact“, also das „Gemeinsame Wirken“, als ein möglicher Denkansatz zur Bewältigung ebendieser Herausforderungen.

Das Collective Impact-Prinzip wurde erstmals im Jahre 2011 von Kania und Kramer formuliert und beschreibt den Gedanken, dass nachhaltige und ganzheitliche Lösungen für gesellschaftliche Probleme nur dann erzielt werden können, wenn gesellschaftliche Akteure ihre Arbeitskraft zur Erreichung eines fest definierten gemeinsamen Ziels bündeln und gezielt zusammenarbeiten (Kania \& Kramer 2011, S. 36 ff.).

Aus diesem zentralen Ansatz heraus hat auch ddn das Collective ImpactPrinzip für seine Netzwerkarbeit übernommen und auf die eigenen Rahmenbedingungen übertragen:

Durch Digitalisierungsschübe, die demografische Herausforderung, zunehmende Diversität, kulturellen Wandel, Fachkräftemangel, die Spaltung unserer Gesellschaft und New Work wird das System Arbeit deutlich berührt und vor bisher unbekannte Probleme gestellt, die aber zugleich neue Chancen eröffnen. Eine zeitgemäße HR-Arbeit an der Schnittstelle von Unternehmen und Gesellschaft nimmt die gemeinsamen Herausforderungen in den Blick, öffnet sich, greift die vielfältigen Facetten des sozialen Wandels auf, nutzt ihn und stärkt den Menschen in ebendieser neuen Arbeitswelt. Personalarbeit ist demnach nicht nur in ihrem betrieblichen Teil Akteur und in der Beziehung zum Umfeld Getriebener, sondern ist wichtiger Teil der Interaktion mit der Gesellschaft. Die Konsequenz ergibt sich darin, dass Unternehmen sich fragen müssen, was der gesellschaftliche Wandel mit ihnen zu tun hat, wie sie auf ihn reagieren oder diesen beeinflussen können. Doch das ist leichter gesagt als getan, denn gemeinsame Probleme können nicht mehr von einzelnen Akteuren allein gelöst werden. Keine Institution - sei es eine staatliche, eine unternehmerische oder zivilgesellschaftliche - ist in der Lage, im Alleingang zu tragfähigen Lösungen zu gelangen. Die Lösung muss vielmehr im gut organisierten Zusammenspiel mehrerer Akteure aus verschiedenen gesellschaftlichen Sektoren liegen, das über ein unverbindliches Netzwerk bewusst hinausgeht. Collective Impact als erfolgreiches Modell der Wirksamkeit ist in diesem Kontext die Selbstverpflichtung wichtiger Akteure auf eine gemeinsame Zielsetzung und ein gemeinsames Vorgehen zur Lösung komplexer Herausforderungen. ddn möchte Motor für diesen Systemwandel sein, indem eine Neuausrichtung entlang der Prinzipien des Collective Impact-Modells angestrebt wird. So wird der bisher praktizierte Ansatz des „Voneinander Lernens" weiter in die ambitionierte Richtung des „Gemeinsamen Wirkens" gedacht. Konkret bedeutet dies, dass nicht mehr nur berücksichtigt werden soll, was Organisationen zukünftig benötigen könnten, sondern 
wie gemeinsam mit anderen Sektoren ein wirkungsvoller Beitrag zum nachhaltigen sozialen Wandel geleistet werden kann.

Wie gestaltet sich diese Erkenntnis nun jedoch in der praktischen Vereinsarbeit? Netzwerkarbeit ist wertvoll, erschöpft sich aber häufig in unverbindlicher Zusammenarbeit. Man betont die Gemeinsamkeiten, verpflichtet sich aber zu nichts. Niemand muss etwas aufgeben, sich an Erfolgen messen lassen oder gar Ressourcen teilen. Das ist praktisch, aber meist wirkungslos. Eine echte Kooperation im Sinne von Collective Impact hingegen verlangt neben Verbindlichkeit das Teilen von Ressourcen. Sie setzt ein gemeinsames Problemverständnis und gemeinsame Zielsetzungen voraus. Gemeinsame Erfolgsindikatoren erlauben es allen Akteuren, ihre Aktivitäten auf die Erreichung der gemeinsamen Ziele auszurichten und ermöglichen ein gemeinsames Lernen aus Erfolgen wie auch aus Misserfolgen. Die Partner ermutigen sich gegenseitig, die jeweiligen Stärken so einzubringen, dass das gemeinsame Anliegen unterstützt wird. So erreichen sie, dass sich konstruktive Aktivitäten im Miteinander verstärken. Eine Kooperation in diesem verstandenen Sinne ist anstrengend, aber dafür effektiv und wirksam. Damit wird ein neues Feld der Personalarbeit eröffnet, da nach dem Collective Impact-Prinzip nun grenzüberschreitende Methoden angewendet werden.

Das Demographie Netzwerk e.V. (ddn) macht es sich zur Aufgabe, auf dem beschriebenen Weg die Brücke zwischen gesellschaftlichen Entwicklungen und unternehmerischem Erfolg zu bauen und gemeinsam das Potenzial neuer Arbeitswelten zu entwickeln.

\section{Neue Herausforderungen für die Zukunft: Netzwerke als Teil der Lösung}

Netzwerke nehmen aus zweierlei Gründen eine Schlüsselrolle in der Bewältigung von gesamtgesellschaftlichen Fragestellungen ein. Auf der einen Seite bedarf es bestimmter Netzwerke in der Funktion als Plattform, auf der Erkenntnisse aus der Wissenschaft für die Praxis aufgeschlüsselt werden können, d.h. ihnen kommt eine entscheidende Transferfunktion zu. So widmen sich Netzwerke der Frage, wie Ideen und Ergebnisse aus der Forschung in konkrete Handlungsempfehlungen für die Praxis übersetzt und diese auf den Unternehmenskontext übertragen werden können. Auf der anderen Seite repräsentieren Netzwerke neue Formen der unternehmensübergreifenden Partizipation, indem sie Akteure verschiedenster Betriebe miteinander vernetzen und in den Dialog bringen. Dies äußert sich einerseits darin, dass Methoden der partizipativen Kommunikation bewusst übernommen werden, beispielsweise das Working Out Loud-Prin- 
zip. Andererseits ist es der zukunftsgerichtete Ansatz, überbetriebliche Lösungen zu entwickeln und somit eine Übertragbarkeit auf den gesamtgesellschaftlichen Kontext zu ermöglichen, der eine gute Netzwerkarbeit ausmacht.

Hierzu werden im ddn folgende drei Leitideen verfolgt - nicht nur in der Netzwerkarbeit, sondern auch in Form eigener Praxisprojekte:

- Überbetriebliches Lernen durch einschlägige Qualifizierungsprogramme

- Überbetriebliche Karriereplanung unter dem Blickwinkel von fresh perspectives ${ }^{1}$

- Überbetriebliche Tätigkeitswechsel

Eine Erkenntnis, die sich aus der Projektarbeit des ddn ergeben hat, ist der notwendige Dialog, der mit der Politik geführt werden muss, um eine erfolgreiche Veränderung von Rahmenbedingungen zu ermöglichen, sodass derartig zukunftsweisende Ansätze in der Praxis realisiert werden können. Ein Leuchtturmprojekt ist in diesem Kontext das BMBF-geförderte Verbundprojekt TErrA ${ }^{2}$, das sich mit dem überbetrieblichen Tätigkeitswechsel in Unternehmensnetzwerken befasste und an dem ddn maßgeblich mitgewirkt hat. TErrA-Ziel ist, die Erwerbsverläufe innerhalb gewisser Branchen präventiv zu gestalten, indem frühzeitige Tätigkeitswechsel von Mitarbeitenden herbeigeführt werden, um so für diese einen geistig wie auch körperlich gesunden Renteneintritt zu gewährleisten. Eine Kernerkenntnis des in den Jahren von 2016-2019 durchgeführten Projektes ist es, dass, nur wenn von Politik und Verwaltung ein grundlegender Rahmen für ebendiese Tätigkeitswechsel geschaffen wird, der den Wechsel auch aus bürokratischer Sicht barrierefrei gestaltet, der Prozess nachhaltig in die Betriebsabläufe integriert werden kann.

Genau an diesem Punkt wird der Bedarf für die Implementierung des Collective Impact-Prinzips sichtbar: Alle gesellschaftlichen Akteure müssen gezielt zusammenarbeiten, um die Herausforderungen des demografischen Wandels zu bewältigen, die Kollaboration im Unternehmenskontext allein ist oftmals nicht ausreichend, um Strategien nachhaltig umzusetzen. Netzwerke verstehen sich in diesem Zusammenhang durch ihre Funktion als „Klammer“ all dieser Akteure als eine Schlüsselfigur. 


\section{Netzwerke der Zukunft: mit offenen Fragen zum Erfolg}

Wie kann gute Netzwerkarbeit zukünftig jedoch so gestaltet werden, dass nicht nur die Bedürfnisse von Unternehmen, sondern die aller gesellschaftlicher Akteure berücksichtigt werden? Auch, um getreu dem Collective Impact-Prinzip handeln zu können? Kollegiales Benchmarking erweist sich im unternehmensübergreifenden Austausch zwar als vielversprechend, doch ist dies im gesamtgesellschaftlichen Austausch nicht zielführend.

An dieser Stelle hat Das Demographie Netzwerk e.V. (ddn) sich bereits deutlich positioniert und Folgendes festgestellt: Die eine Antwort gibt es nicht. Nur kreatives Denken und der kollektive Diskurs können den Herausforderungen der Zukunft gerecht werden. Nicht zuletzt die Auswirkungen der Corona-Pandemie haben verdeutlicht, dass starre Lösungsstrategien und ein Handeln nach „Schema F“ oft eher eine Barriere darstellen, als dass sie Sicherheit geben.

An diesem Punkt kann ein offeneres Konzept zum Tragen kommen: Das von Pörksen (2020) entwickelte „Prinzip der offenen Frage“ kann als Hilfestellung dienen, wenn zu schematische Methoden-Ansätze nicht mehr weiterführen. Pörksen (2020) konstatiert, dass „,...) die meisten Fragen, die gestellt werden, eigentlich gar keine sind, weil der angeblich Fragende die Antwort schon kennt" (S. 50). Eine offene Frage lässt sich somit als eine Frage verstehen, deren Antwort bisher noch niemand kennt. Dies macht sie einerseits zu einem machtvollen Instrument, bedeutet aber auch, sich auf unbekanntes Terrain hinauszuwagen und sich auf die eigene Unsicherheit einzulassen. Ein Prozess, der sicherlich mit Irritationen verbunden ist. Gerade diese Phase ist für Unternehmen oft ein Hindernis, welches es zu überwinden gilt, um in die pragmatische Lösungssuche einzusteigen. An diesem Punkt kommen erneut die Netzwerke zu Hilfe, denn diese können beispielsweise durch moderierte Austauschformate dazu beitragen, diese Hürden zu überwinden und den sprichwörtlichen gordischen Knoten zu durchtrennen.

Durch gezieltes Handeln von Netzwerken, die Unternehmen strukturelle Angebote machen, sich den gesellschaftlich relevanten Aspekten der Personalarbeit zu widmen, können nach dem Prinzip der offenen Frage Themen bearbeitet werden, die von einer klassischen Personalabteilung nicht gelöst werden können. Und gerade an diesem Punkt möchte ddn als zukunftsgerichtetes Netzwerk seine gesellschaftliche Funktion als „Klammer der Akteure" wahrnehmen und sich durch die Schaffung innovativer Formate positionieren. Sei es in Form von Erzählsalons, Barcamps oder Austauschforen, hiermit sollen gezielt Akteure unterschiedlichster Sekto- 
ren zusammengebracht werden, um genau die „offenen“ Fragen zu stellen, die zum jetzigen Moment noch keiner zu beantworten vermag.

\section{Fazit}

Der vorliegende Beitrag hat verdeutlicht, was von einem Netzwerk der Zukunft verlangt wird: Beweglichkeit, wo sonst Starre herrscht, Flexibilität sowie Kreativität, wo andere zu schematisch denken. Die gesellschaftliche Verantwortung von Netzwerken liegt in der Anregung und Moderation eines übergeordneten Diskurses und dem Transfer von wissenschaftlichen Erkenntnissen in die konkrete Praxis. Um dies gewährleisten können, sind Netzwerke auf die Unterstützung von der öffentlichen Hand dringend angewiesen. Viele Netzwerke finanzieren sich fast ausschließlich durch Mitgliedsbeiträge oder tragen sich von der einen in die nächste Projektförderung. Eine strukturelle Förderung von Netzwerken würde dazu beitragen, Netzwerkarbeit zu professionalisieren und noch zielgerichteter wirken zu lassen.

\section{Literatur}

Anzinger, R. (2006): Begrüßung zur Gründungsveranstaltung des DemographieNetzwerkes (ddn). Rede vom 27.3.2006 im Bundesministerium für Arbeit und Soziales, Kleisthaus K1.

Botthof, A.; Hartmann, E. A. (Hrsg.) (2015): Zukunft der Arbeit in Industrie 4.0. Wiesbaden: Springer Vieweg.

Fleck, T.; Krauß, B. (2011): Kollegiales Benchmarking. In: Bundesanstalt für Arbeitsschutz und Arbeitsmedizin (Hrsg.): Qualitätssicherung in der Demografieberatung. Bielefeld: Bertelsmann Verlag. S. 31-43.

Kania, J.; Kramer, M. (2011): Collective Impact. In: Stanford Social Innovation Review. Verfügbar unter: https://ssir.org/?ACT=219\&lv=QriLzY9tzCP6LkkeAtyiEc HsOwVLiV55CozcJBIIQcz1\%2BPPjRUo8K0GT8ZEaS49G9UN1V1dgt8WHlbv Zm9xmEZh3coC06P2cCxD1D17ca9Sv76nG0PwK5fUJaopQLow1S0fRuwIkCq1 sWGPgnjDAzA\%3D\%3D.

Marquardt, T.; Klopprogge, A.; Deller, J. (2019): Grenzverkehr. Wie Personalarbeit die Brücke zwischen unternehmerischem Erfolg und gesellschaftlichen Entwicklungen bauen kann. In: Goinger Kreis e.V. (Hrsg.): Grenzüberschreitungen zwischen Unternehmen und Gesellschaft. Herausforderungen im System Arbeit gemeinsam bewältigen. Hohenwarsleben: VAS - Verlag für Akademische Schriften. S. $11-31$.

Pörksen, B. (2020): Fragen, die alles verändern. In: Die Zeit. 20.8.2020. S. 50. 
RAG Stiftung (o.D.): Megatrends. Verfügbar unter: https://www.glueckauf-zukunft .de/zukunftsstudie/methodik-und-vorgehen/megatrends/.

Statistisches Bundesamt (Hrsg.) (2006): Bevölkerung Deutschlands bis 2050. 11. koordinierte Bevölkerungsvorausberechnung. Verfügbar unter: https://www.destat is.de/DE/Themen/Gesellschaft-Umwelt/Bevoelkerung/Bevoelkerungsvorausbere chnung/Publikationen/Downloads-Vorausberechnung/bevoelkerung-deutschlan d-2050-presse-5124204069004.pdf?_blob=publicationFile.

\section{Referenzen}

1 Damit ist hier die Perspektive von neu eingestellten Mitarbeitenden auf bestimmte betriebliche Aspekte gemeint, von der ein Unternehmen profitieren kann, da diese einer sogenannten „Betriebsblindheit“ entgegenwirken kann.

2 TErrA = Tätigkeitswechsel zum Erhalt der Arbeitsfähigkeit. 
